\title{
Nursing care for patients with the Ehlers-Danlos and other hypermobility syndromes
}

\author{
Margrieth Veenhuizen, Lidwien Cornelissens, Maria Blackmon \\ Rijnlandziekenhuis, Leiderdorp, Netherlands. \\ Correspondence: Margrieth Veenhuizen. Address: Amstelstraat 8a, 3522 CR Utrecht, the Netherlands. Email: \\ mveenhuizen@telfort.nl
}

Received: J une 19, 2013

DOI : $10.5430 /$ jnep.v4n4p52

\section{Abstract}

Ehlers-Danlos syndrome is a rare genetic disorder, with mainly three symptoms: hypermobility, fragile skin and fragile veins. In this article we discuss the syndrome and we consider the consequences for nursing care of the three most common types of EDS. Starting point are the health patterns of Gordon in combination with peri-operative situations.

Ehlers-Danlos syndrome (EDS) comprises a clinically and genetically heterogeneous group of inherited connective tissue disorders, which are mainly characterized by a variable degree of generalized joint hypermobility, skin hyperextensibility, easy bruising and skin fragility.

Two facts have to be taken into consideration: firstly, the severity of the manifestation of the syndrome varies among patients, even among members of the same family. It is not possible to make sharp distinctions based on clinical findings between the types. Secondly, it is impossible to clearly distinguish the different types based on clinical findings, as the syndrome manifests itself in many different ways. So far, there are no simple and reliable clinical tests or laboratory test.

Furthermore, patients often have additional problems, which are not characteristics of the known types of Ehlers Danlos. As a nurse it is important to be aware of the strongly varying needs of patients both during the day and night and their pain and fatigues, which all patients report, and report as being as very troublesome. It is essential that nurses, during hospitalization, should bear in mind that patients may have to cope with hypermobility of the joints, fragile skin, possible dysfunctional vasoconstriction and the often present generalized autonomous disregulation. Because the pattern of complaints varies markedly from patient to patient, good communication between nurse and patient is very important.

\section{Key words}

Joint hypermobility, Skin hyperextensibility, Bruising skin, Skin fragility, Ehlers Danlos syndrome, Hypermobility syndrome

\section{I ntroduction}

At admission to a hospital, a patient usually knows less about his or her illness than the department nurse. However, the reverse is often the case for patients with the Ehlers-Danlos syndrome (EDS). There are two reasons for this. First, this syndrome is so uncommon that a nurse will probably never have had to deal with it before and will therefore recognize neither the syndrome nor the symptoms. Second, for many patients, the diagnostic process for EDS is so long and difficult 
that, during this time, they will have studied their own symptoms and have discovered the collagen disorder for themselves ${ }^{[1]}$.

More than many other illnesses, EDS is a disorder that is different for each patient. The specific nature of this disorder requires great dedication on the part of both the nurse or nursing staff and the patient. The nurse must listen professionally and critically to the patient if they are to work together well. The patient can be given, if possible, time to explain what EDS is to the nurse and must, in particular, explain its specific consequences for him/her as a patient.

The aim of this article is to clarify the nursing procedures required for these disorders and to explain how a nurse can best deal with a patient with EDS or other hypermobility syndrome who is admitted to hospital. Throughout the text the term EDS may indicate EDS or other hypermobility syndrome, if the topic is generalized hypermobility.

\section{Types of EDS}

At least six types of EDS can be distinguished. These types share a number of symptoms. Only three of the most common types will be discussed in this article: the classical, hypermobility and vascular types, which together account for $90 \%$ of the prevailing EDS cases. However, to a greater or lesser degree, hypermobile joints, highly elastic skin and bruising are common to all types. Table 1 shows the extent to which these 3 problems occur among the six types of EDS.

Table 1. Magnitude of the three main problems associated with the most common types of EDS*

\begin{tabular}{llll}
\hline Types of EDS & Highly elastic skin & Hypermobile joints & Frailty of vessels and organs \\
\hline Classical type & +++ & + & + \\
Hypermobility type & + & +++ & + \\
Vascular type & + & + & +++ \\
Kyphoscoliosis type & + & ++ & + \\
Arthrochalasia type & ++ & +++ & ++ \\
Dermatosparaxis type & ++ & +++ & + \\
\hline
\end{tabular}

* + limited ; ++ moderate ; +++ severe impact; benign joint hypermobility syndrome resembles EDS hypermobility type

\section{The medical history taking}

A number of problems and issues relating to the syndrome require attention when admitting an EDS patient to hospital for a procedure or operation. These play a role in addition to the indication for admission and should be included in the history taken by the nurse. To a greater or lesser degree, there are four prominent chronic problems ${ }^{[2]}$ :

1) Hypermobile joints, possibly with (sub)luxation or dislocation as a result;

2) Highly elastic, fragile skin (where serious injury can result from limited trauma);

3) Skin which bruises easily (hematoma) because the connective tissue around the blood vessels is less strong;

4) In EDS of the vascular type, the connective tissue within the blood vessel walls and the hollow organs (intestines, heart, etc.) is weak, resulting in a high risk of bleeding and ruptures. In the other types of EDS, the connective tissue around the blood vessel walls is less tight, resulting in an increased risk of hematomas ${ }^{[1 \mathrm{p} 94]}$.

For this complex disorder, which may involve many organs and organ systems, a thorough medical history taking from each individual with EDS is of the utmost importance. Thereafter, a specific nursing plan can be drawn up. For a patient with EDS, the areas which initially require nursing care will emerge from the medical history.

While taking the history, the nurse will integrate the 11 health patterns (see Table 2) with EDS-specific issues and the individual's specific problems. When designing a specific nursing plan, the basic assumptions are as follows. 
Table 2. Classification of 11 functional health patterns ${ }^{[3]}$

\begin{tabular}{|ll|}
\hline 1) & Health perception - health management pattern \\
3) & Elimitional - metabolic pattern \\
4) & Activity - exercise pattern \\
5) & Sleep - rest pattern \\
6) & Cognitive - perceptual pattern \\
7) & Self-perception pattern \\
8) & Role relationship pattern \\
9) & Sexuality - reproductive pattern \\
10) & Coping - stress intolerance pattern \\
11) & Values - belief pattern \\
\hline
\end{tabular}

Each patient experiences medical problems differently, and each one tries to maintain mental and physical health in his or her own way ${ }^{[4]}$. A deteriorating condition such as EDS can severely impair mental well-being and physical functioning.

Activity patterns are partially determined by the degree of pain and fatigue experienced. Although thinking about symptoms and the effect of exercise has changed greatly in recent years - for many disorders, symptoms are reduced by exercise - it is very important that EDS patients should carefully alternate rest and exertion. The patients themselves generally know their individual 'instructions for use' well ${ }^{[3]}$.

Regarding dietary and metabolic patterns, factors such as diet, stomach and intestinal pain, constipation or defects such as a rectum prolapse will influence how a patient deals with the problems. Each patient experiences, accepts and deals with the burden of a chronic disorder differently. When nursing a patient with EDS, it is important to determine the nature and seriousness of the symptoms. This applies both to the symptom-related problems as well as to the problems that have led to hospitalization. It is also important to bear in mind that the patient's condition may vary greatly each day or even throughout the day. It is not unusual for a patient to be able to perform all the normal daily activities independently in the morning and to need help and assistance to change clothing in the evening.

\section{Case study}

A 45-year-old woman checks in at the gynecology department in order to be admitted for the removal of her uterus via the abdominal wall (abdominal uterus extirpation). When the patient is greeted upon arrival, the nurse shaking the patient's hand notices that it feels 'strange', like a velvet flannel with bones. The patient has EDS classical type. The skin of patients with this type of EDS feels velvety soft and doughy.

Notably the patient has taken along her own mattress and pillow because she is unable to lie on hard hospital beds because of neck and back pain resulting from hypermobile joints. She explains that for her type of EDS, hypermobility, primarily of the smaller joints such as those of fingers, ribs and the wrists, play an important role. She is chronically constipated and frequently has intestinal complaints.

To ensure that the operation, hospitalization, discharge and convalescence of an EDS patient are as successful as possible, it is important that a thorough medical history is taken. There follows a description of how the process of admission, pre-operative screening, pre-, per- and post-operative support, convalescence and discharge can be controlled to the optimum.

\section{Admission}

The issues requiring extra attention when taking the medical history are: 


\section{Use of medicine}

In many cases, the patient will be using various medicines, some on demand, not regularly, such as pain killers. Will the patient also be responsible for managing these on the ward? Make sure there are clear arrangements about this, particularly in relation to the department's prevailing policy on pain control. If a patient is already using painkillers, the department's policy may need to be adapted to this to prevent the patient either from becoming overdosed or from having to change to a medication cited in the formulary.

\section{Allergies}

Besides any allergies, most EDS patients are hypersensitive to plasters. In addition, sometimes when sticky plaster is removed, part or all of the epidermis is torn. In such cases, other solutions for affixing bandages must be sought, such as elastic bandages or compresses.

\section{Eating habits}

It is not unusual for a patient to have an adapted diet because of sensitivity or intolerance of the intestines or a hypotonic gastrointestinal tract. There is a higher risk of constipation as a result of lengthy bed rest. You and the patient should keep an eye on this; if necessary, ask a dietician to set up a diet enriched with fibre and with plenty of liquids.

Many EDS patients suffer from a motility disorder in the gastrointestinal tract and use medication for this. Narcotics and bed rest can increase these problems. You and the patient should keep an eye on this and take timely action, for instance by taking measures such as the use of lactulose and a micro enema, combined with the avoidance of medication known to aggravate these problems ${ }^{[5]}$.

\section{Pre-operative screening}

The following issues are of importance:

- Does the patient have problems with moving his or her head or opening his or her mouth?

- Predicting problems at intubation.

When anaesthetizing such patients, a number of precautions should be kept in mind. When a patient is under anesthesia, the muscles relax; in EDS patients these muscles help to stabilize the hypermobile joints. Thus, there is an increased risk of joint (sub)luxation. The patient must therefore be moved with the utmost care. The anaesthesiologist should also carefully monitor the patient's maxillary joints while inserting a tube into his or her throat. Also these joints can easily become luxated. The risk of a headache after the operation is reduced by repositioning of the jaws and removing the tube as quickly as possible if this occurs ${ }^{[6]}$.

- Does inserting a needle to draw blood or connect a drip prove to be problematic?

The nurse or doctor should be aware of the fact that the patient's vessels are fragile and tend to roll away from the tip of the needle. The vessels do not contract well either, or the collagen around the vessels is less tight, increasing the chances of serious haematomas. Huge bruises can result from a misplaced injection or needle insertion because the tissue around the vessels is less firm. It is advisable to ask someone with a great deal of experience with injections or needle insertion to perform this task, to prevent extra pain and injury.

\section{Pre-, per- and postoperative support}

- In addition to the acute pain brought on by the operation, EDS patients also may experience chronic pain in various places in the body. This is the result of hypermobile and sometimes damaged joints, joint capsules and muscles. It is advisable to discuss the possibilities of pharmacological pain management before surgery and to call a physiotherapist early on after surgery for support during the often slow convalescence ${ }^{[7]}$. 
- There is a higher risk of postoperative sleeplessness as a result of the pain caused by the operation in addition to the already existing chronic pain symptoms caused by EDS. The patient and nurse can discuss how best to deal with this problem. Lying in bed for long periods often leads to back pain. The patient may require more exercise that the standard operation protocol dictates. However, if the patient is suffering from complaints of the back and pelvis, resulting from lying on a hard operation table, it may not be possible to follow the mobilization protocol because a few days of bed rest are needed before mobilization can be initiated ${ }^{[8]}$.

- Due to the patient's highly fragile skin, there is an increased risk of decubitus ulcers (bedsores, pressure ulcers). On medical grounds alone, the ED syndrome calls for an anti-decubitus mattress. In the case above, the patient took her own pillow along (remember the risk of subluxation of the maxillary joints) to enhance her comfort when lying down and sleeping. If the patient is bed bound, a dietician can prescribe a diet high in protein. Bedsores can be prevented by changing positions, through massage and by mobilizing the patient as early and as much as possible.

- It is important to monitor bleeding of the incisions. Due to poor quality of vessel walls, poor vessel contraction and possibly bleeding disorders, the risk of continued bleeding after the operation is higher.

- Total floppiness (hypotonia) of the organ walls is an important issue to watch for. In cases of an abdominal injury, a spica elastic bandage can be applied to prevent further bleeding.

Among patients with the vascular type, vessel and organ problems will be prominent far more often than normal. Upon meeting patients with this vascular type, one will notice the thin transparent skin. Sometimes (certainly not always) people with this type of EDS have characteristic facial features such as a pointed noise, hollow eyes, missing earlobes and epicanthic eyelids. More often they have an aged aspect of the hands (acrogeria). The risk of the vessel and organ walls tearing is greatly increased among this (small) group of patients. Because the contact between the nurse and patient is close at the ward, the nurse will be the first to notice the symptoms of these complications; she or he should take action quickly. Although it is generally wise to have an intensive care bed available for EDS patients, it is a prerequisite for surgery upon patients with the vascular type.

\section{Convalescence and discharge}

Partly due to the issues for each individual patient, it is difficult to make any generalized judgment about the extent and intensity of an EDS patient's convalescence or what help may be required at home after discharge. The individual's need for further care will have to do with factors such as the number of days which have elapsed since the surgery took place, the patient's condition and ability to manage independently prior to the operation and the extent of pain and disability.

A slow recovery and extended convalescence period often have consequences for the duration of the hospitalization. In order not to extend this time any more than needs be, it is important to give timely attention to the issue of whether homecare and possible other resources have to be provided or not ${ }^{[4]}$.

\section{Summary}

The relatively rare Ehlers-Danlos syndrome is an unknown disorder to many nurses. Although there are differences in symptoms and degrees of seriousness, three problems occur among patients to a greater or lesser degree: 1) hypermobile joints, 2) highly elastic, fragile skin and 3) easy bruising. A nurse who has to take care of a patient with EDS will have to gain some knowledge of the symptoms and can, through the nursing diagnosis, determine the range of problems as well as the related contributory factors and consequences. By doing so, he or she can prevent a number of complications and also reduce their seriousness. 


\section{References}

[1] Jacobs.JWG, Cornelissens LJM, Veenhuizen MC, Geenen R, Ehlers Danlos Syndroom, Een multidisciplinaire benadering (Ehlers Danlos Syndrome, multidisciplinairy team), Boom Amsterdam, 2009.

[2] Arets J, Vaessen J, Professionele verpleegkunde deel 1 (Professional Nursing Care part 1). Leiden: Spruyt, van Mantgem en de Does, 1993.

[3] Gordon M, Manual of nursing diagnosis. Maarssen: Elsevier/ de Tijdstroom. 1998; 2: 97-98.

[4] Gordon M, Manual of nursing diagnosis 1, Utrecht: Lema, 1998.

[5] McCloskey JC, Bulecheck GM, Nursing interventions classifications. Utrecht: De Tijdstroom, 1997.

[6] Ainsworth SR, Aulicino PL. A survey of patients with Ehlers-Danlos syndrome. Clin Orthop. 1993; 286: 250-6.

[7] Bos A, Leeuwen RR van, Rijswijk RH van, Gent W van, Hoekstra T, Bos DG van de, Verplegen van zorgvragers met een psychiatrische ziekte. (Nursing care of psychiatric clients) Utrecht: Thieme Meulenhoff, 2002.

[8] Carpentino LJ, Nursing diagnosis, application to clinical practice. Philadelphia: Lippincott, 2000. 